\title{
Group work in a nursing curriculum: A teaching strategy to enhance student engagement
}

\author{
E Mukurunge, ${ }^{1} \mathrm{MNsg} ; \mathrm{L}$ Badlangana, ${ }^{2} \mathrm{PhD} ; \mathrm{C}$ N Nyoni, ${ }^{3} \mathrm{PhD}$ \\ ${ }^{1}$ Paray School of Nursing, Thaba Tseka, Lesotho \\ ${ }^{2}$ Department of Biomedical Sciences, Faculty of Medicine, University of Botswana, Gaborone, Botswana \\ ${ }^{3}$ School of Nursing, Faculty of Health Sciences, University of the Free State, Bloemfontein, South Africa
}

Corresponding author: E Mukurunge (evamukurunge@gmail.com)

\section{Why was the idea necessary (problem)?}

Health professions education institutions across the globe are adopting competency-based education (CBE) models. ${ }^{[1]}$ Inherent in CBE is learner centredness, which emphasises the contribution of learners to the learning process. Nursing education institutions (NEIs) in Lesotho adopted CBE for all undergraduate nursing programmes, inspired by the Government's need for improved quality of learning that aligns with global trends in health professions education. ${ }^{[2]}$ Nurse educators from NEIs who adopted CBE use group work as a dominant teaching strategy to enhance learner centredness. Group work, when appropriately applied, is associated with enhanced teamwork, motivation and deep learning. ${ }^{[3]}$ Nursing students in NEIs in Lesotho asynchronously prepare for class activities through engaging with specified learning resources, such as study guides and workbooks. Nurse educators moderate learning in class and randomly assign students to small groups. The use of random allocation is deliberate to prepare them for the authentic clinical environment, where there is no autonomy in choosing team members. As a result, differences in learning styles, personalities and experiences were not taken into account. Each group collectively solves a specific problem, applying knowledge learnt during the asynchronous time. However, educators observed poor engagement, dominance by some students and general disparities in the performance among the groups. Therefore, in view of these challenges, we explored how the students experienced group work.

\section{What was tried (approach)?}

Student nurses' $(N=30)$ experiences of group work were explored through qualitative research. These student nurses were enrolled in a competencybased nursing programme and had experienced group work in different modules, which were facilitated by various nurse educators. Data were collected by a research assistant, who facilitated two focus group discussions using a semi-structured interview guide. Data were analysed using Creswell's method of thematic analysis. ${ }^{[4]}$

Ethical approval was granted by the Ministry of Health, Lesotho (ref. no. 68-2018) and all participants gave informed consent.

\section{What were the lessons learnt (outcomes)?}

Participants thought that their diverse personalities influenced the functionalities of groups. In the initial stages of group work, time was spent on understanding the personalities of the group members, leaving little time to meet the specified learning outcomes. The establishment of leadership seemed to enhance group cohesion and minimised conflicts. However, participants expressed the need for a structured group conflict-resolution strategy. Participants expected nurse educators to frequently reward the best performing groups as a means of motivating all groups to perform better.

\section{Conclusions}

Nursing programmes are being modified to provide quality learning experiences for students. Group work has the potential to improve the learning experience of nursing students in CBE. Nonetheless, factors that negatively affect group work need to be addressed to maximise the benefits. Therefore, nurse educators need to apply innovative approaches to motivate collaboration in the groups, such as game-based learning, and establish appropriate strategies to ensure leadership and conflict resolution.

\section{Declaration. None.}

Acknowledgements. The authors acknowledge Dr M Gonzaga (Makerere University, Uganda), Dr N Mannathoko (University of Botswana) and Dr W Cordier (University of Pretoria) for their expert inputs and for critically reading this manuscript. The subSaharan Africa-FAIMER Regional Institute (SAFRI) is acknowledged for providing the platform for incubating and developing this project.

Author contributions. All authors contributed to the conceptualisation, design and execution of this study. All authors equally contributed to the writing of the article. Funding. None.

Conflicts of interest. None.

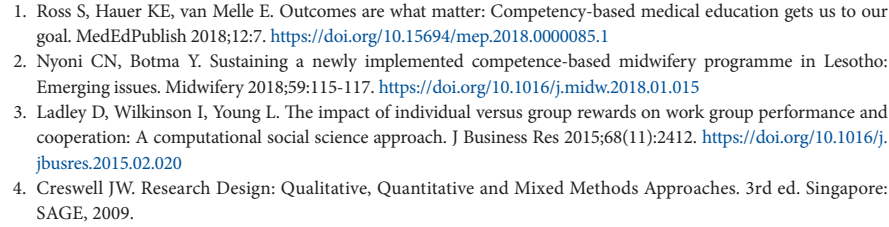

3. Ladley D, Wilkinson I, Young L. The impact of individual versus group rewards on work group performance and cooperation: A computational social science approach. J Business Res 2015;68(11):2412. https://doi.org/10.1016/j. jbusres.2015.02.020

4. Creswell JW. Research Design: Qualitative, Quantitative and Mixed Methods Approaches. 3rd ed. Singapore SAGE, 2009.

Accepted 29 June 2020.

Afr J Health Professions Educ 2020;12(3):95. https://doi.org/10.7196/AJHPE.2020.v12i3.1358 